\title{
Resección abdominoperineal isquioanal con reconstrucción de pared posterior de vagina con colgajo miocutáneo de músculo recto abdominal vertical
}

\author{
(Ischioanal abdominoperineal resection with reconstruction of posterior wall of vagina \\ with vertical rectus abdominis myocutaneous flap
}

José P. Rivera-Chavarría, ${ }^{1}$ José E. Murillo-Rodriguez ${ }^{1}$ y Alfredo Vargas-Villalobos ${ }^{2}$

\begin{abstract}
Resumen
El colgajo del recto abdominal miocutáneo vertical, es un excelente colgajo que rellena el gran defecto en la pelvis luego de una resección abdominoperineal isquioanal, siendo libre de tensión y reduciendo significativamente la incidencia de complicaciones de la herida perineal y la estancia hospitalaria, con el cual se puede reconstruir la pared posterior de la vagina. Se reporta aquí el primer caso en Costa Rica de una resección abdominoperineal isquioanal, con reconstrucción de pared posterior de vagina con colgajo del recto abdominal miocutáneo vertical, practicada con éxito en una paciente de 62 años, con un adenocarcinoma rectal localmente avanzado con infiltración de pared posterior de vagina.
\end{abstract}

Descriptores: tumoración rectal, exanteración pélvica, resección abdominoperineal, colgajo miocutáneo de músculo recto abdominal vertical.

\section{Abstract \\ Vertical rectus abdominis myocutaneous flap, is an excellent flap that fills the large defect in the pelvis after an adominoperineal ischioanal resection; it is free from tension, significantly reduces the incidence of perineal wound complications and hospital stay and can be used to reconstruct the posterior wall of the vagina. We report the first case of Costa Rica abdominoperineal ischioanal}

Cirugía y trabajo realizados en conjunto entre la Unidad de Colon y Recto del Servicio de Cirugía General y el Servicio de Cirugía Plástica y Reconstructiva del Hospital México

Afiliación de los autores: "Unidad de Cirugía de Colon y Recto. Servicio de Cirugía General. ${ }^{2}$ Servicio de Cirugía Plástica y Reconstructiva. Hospital México, Caja Costarricense de Seguro Social, San José, Costa Rica.

凶joseriverach@gmail.com resection with reconstruction of posterior vaginal wall with vertical rectus abdominis myocutaneous flap, successfully practiced in a patient of 62 years with locally advanced rectal adenocarcinoma infiltrating posterior wall of the vagina.

Keywords: Rectal tumor, pelvic exenteration, abdominoperineal resection, vertical rectus abdominis myocutaneous flap.

Fecha recibido: 20 de abril 2015

Fecha aprobado: 13 de agosto 2015

Durante la resección abdominoperineal (RAP) extraelevadora o isquioanal, se crea un gran espacio muerto de la pelvis, que puede conducir a hematoma pélvico y herniación del intestino delgado, que afecta negativamente la cicatrización de heridas. ${ }^{1,2} \mathrm{La}$ quimiorradioterapia preoperatoria reduce al mínimo la recurrencia local, pero hace que los tejidos sean más frágiles y se asocia con un mayor riesgo de complicaciones de la herida. ${ }^{1,3} \mathrm{~A}$ su vez, la exanteración pélvica puede ser la única forma de tratamiento para el cáncer rectal primario localmente avanzado o recurrente, que invade estructuras vecinas y que deben ser resecadas para lograr una resección R0. Dicho procedimiento se acompaña de una morbimortalidad significativa, asociada de manera directa a complicaciones de la herida, como dehiscencia y sepsis. ${ }^{4}$

El cierre directo de la herida perineal se asocia con una alta incidencia de complicaciones de la herida con dehiscencia, abscesos perianales crónicos $(37 \%),{ }^{5}$ fístulas y la cicatrización retardada, que ocurre en un $18-70 \%$ de los casos. ${ }^{1,3,4,6}$ Estas complicaciones se relacionan con la presencia de un espacio muerto grande, pobre vascularidad del tejido circundante irradiado, uso de piel irradiada en el cierre, y la contaminación bacteriana debido a la resección del intestino. ${ }^{7}$ Otra de las complicaciones que se observan en los pacientes operados luego de quimiorradiación y se asocian sobre todo a la herida perineal, aparte de lo antes mencionado, es la dificultad para reconstruir la vagina. ${ }^{8}$ Debido a esto, en los últimos años se han propuesto múltiples métodos con el fin de disminuir dichas complicaciones producto del cierre primario.

\section{Caso Clínico}

Paciente femenina de 62 años, con antecedente de bocio multinodular. Consulta con historia de 3 años de evolución 


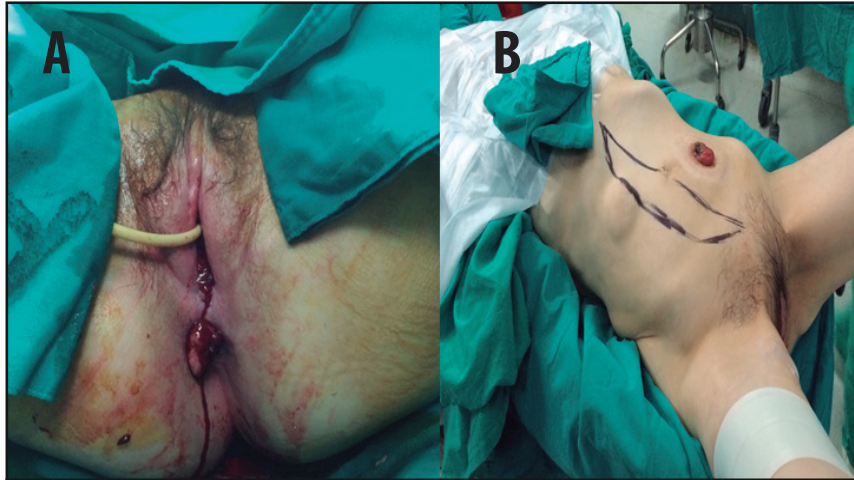

Figura 1. 1A: Tumoración rectal con franca infiltración vaginal. 1B: Marcaje prequirúrgico de colgajo miocutáneo de músculo recto abdominal vertical (VRAM)

de alteración en el hábito defecatorio, asociando 3 semanas de evolución de salida de heces por vagina. Al examen físico se evidencia tumoración anterior de unos $5 \mathrm{~cm}$ de diámetro que compromete ano y pared posterior de vagina (fístula rectovaginal). Se realiza colonoscopia completa que muestra: "carcinoma ano-rectal de $5 \mathrm{~cm}$ en pared anterior", cuya biopsia evidencia: "adenocarcinoma probablemente invasor". El TAC de Cuello-toraco-abdonimo-pélvico reporta: "bocio multinodular con proyección intratorácica, tórax sin lesiones, no presencia líquido libre, hígado, bazo, páncreas, riñones y suprarrenales sin lesiones, engrosamiento ano-rectal en una longitud de $78 \mathrm{~mm}$, con múltiples adenopatías perilesionales". Se realiza una resonancia magnética que reporta: "masa infiltrante mamelonada que afecta el tercio inferior del recto y que impresiona afectar el esfínter del ano, con múltiples adenopatías, lesión parece contactar con la pared posteroinferior de la vagina, existiendo imagen sugestiva de un trayecto fistuloso en el aspecto posterior derecho entre la vagina inferior y el recto distal". Se lleva a quirófano donde se realiza colostomía en asa de colon sigmoides. Luego de esto completa terapia neoadyuvante con radioterapia y quimioterapia. De seguido, la paciente es llevada nuevamente a quirófano, donde se evidencia tumoración exofítica que compromete

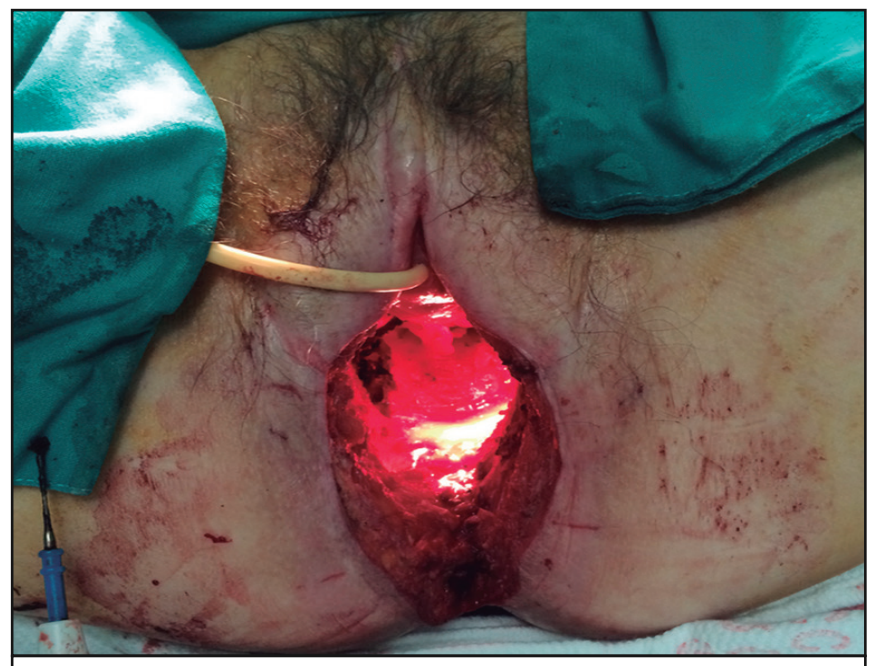

Figura 2. Defecto remanente luego de pélvico-cutáneo tras resección en bloque de tumoración rectal con compromiso de pared posterior de vagina piel perianal y tercio medio e inferior de vagina (Figura 1A). Se realiza marcaje de colgajo miocutáneo de músculo recto abdominal vertical (VRAM) (Figura 1B) y se ejecuta resección abdominoperineal tipo isquioanal, con resección en bloque de pared posterior de vagina (Figura 2). Por último, se realiza colgajo VRAM, utilizándolo para fabricar nueva pared posterior de vagina y rellenar el defecto pélvico dejado por la resección (Figura 3). Paciente con excelente evolución clínica es dada de alta del hospital al postoperatorio siete; no presenta ninguna complicación postoperatoria, con colgajo exitoso. El reporte final de patología muestra: "Adenocarcinoma moderadamente diferenciado de canal anal de $7 \mathrm{~cm}$ de diámetro mayor, que atraviesa músculo esfínter externo, con extensión a piel perianal y a vagina. Invasión vascular, linfática y perineural presentes. Márgenes de resección sin infiltración neoplásica. Uno de 13 ganglios linfáticos perirectales con metástasis de adenocarcinoma (1/13). pT4bN1".

\section{Discusión}

Uno de los métodos más antiguos y utilizados para disminuir las complicaciones del cierre primario es la omentoplastía, la cual ha demostrado que mejora la cicatrización primaria, pero el fracaso de la cicatrización primaria, incluso en la mejor serie, puede ser tan alta como un 10-20\%. ${ }^{1,4,6,9}$

En los últimos años, algunos grupos han reportado el uso de malla biológica para cerrar el defecto perineal, aunque la principal crítica a este método es que no se ocluye el espacio muerto en la pelvis. ${ }^{1,4}$

Para el cierre adecuado de esos tumores del periné que requieren de una extensa resección a menudo en un campo irradiado, se precisa un colgajo versátil, de buen volumen y vascularidad, necesario para llenar el espacio muerto, dar soporte

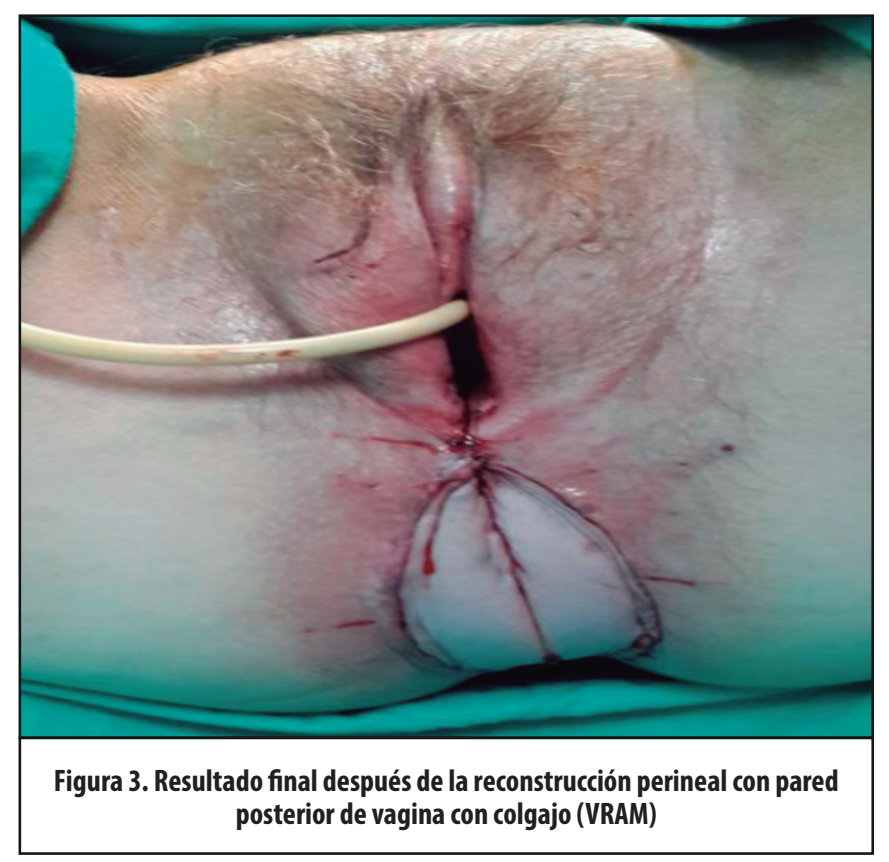


a las vísceras, proveer una adecuada durabilidad de la superficie y que cuando se indique, sirva para reconstrucción vaginal. ${ }^{10}$ Se han descrito varios tipos de colgajos para la reconstrucción de defectos perineales y pélvicos; su utilización ha demostrado que resulta en un menor número de complicaciones de la herida que los métodos de cierre primario. ${ }^{4}$ Entre los colgajos descritos se encuentran: colgajo de gracilis, colgajo de glúteo mayor, colgajo de la arteria perforante glútea inferior (IGAP); sin embargo, el colgajo del recto abdominal miocutáneo vertical (VRAM por sus siglas en inglés), basado en el pedículo vascular epigástrico inferior, cumple con estos criterios y se ha descrito su uso de forma segura y exitosa para la reconstrucción perineal. ${ }^{1,2,11,12}$

E1 VRAM es un método que se conoce desde hace muchos años y que fue introducido por primera vez en 1984, como un reporte de caso por Shukla y Hughes. ${ }^{4}$ El colgajo VRAM llena fácilmente el gran defecto en la pelvis luego de una RAP, llenándola de forma casi completa o completa, libre de tensión, siendo esta la principal ventaja con respecto a los otros colgajos pediculados, que solo rellenan una pequeña proporción del volumen total de la pelvis. Con esto se previene también la herniación del intestino delgado. ${ }^{1}$

Estudios grandes, del uso primario de un colgajo VRAM en RAP por cáncer anal, han demostrado que lograr una larga sobrevida y baja morbilidad perineal, es posible con una resección $\mathrm{R} 0$, con este tipo de abordaje y reconstrucción. ${ }^{2}$

Varios estudios han demostrado que el VRAM reduce la incidencia de dehiscencia de la herida perineal y absceso perineal, sin aumentar las complicaciones de la herida abdominal, en comparación con el cierre primario.?

Cuando la cirugía contempla la extirpación «en bloque» del tumor, incluyendo con frecuencia la pared posterior de la vagina, el VRAM es una excelente opción, pues se puede crear una nueva vagina. ${ }^{1,3,5,8}$

Una desventaja potencial de los colgajos VRAM es el debilitamiento de la pared abdominal. Algunos estudios reportan una incidencia de un 3\% a un 19\% de hernia insicional, por lo que algunos autores recomiendan el uso de una malla para cubrir el defecto dejado durante el VRAM. ${ }^{4,5}$ Con miras de reducir o evitar estas complicaciones de zona donadora abdominal, se ha descrito la técnica de preservación de fascia, separación de componentes y malla en sitio donador, como modificaciones para disminuir dicha morbilidad de zona donadora. Si la fascia es de poca calidad, se evidencia un saco herniario adyacente, o se trabaja en una fascia afectada por cicatrices de procedimientos anteriores; se recomienda la colocación de malla para un cierre sin tensión del sitio donador. Existe una preferencia por la malla biológica sobre la sintética, en términos de un menor índice de complicaciones, sobre todo cuando ha habido contaminación bacteriana por resección intestinal concomitante o colostomía, como suele requerirse en las intervenciones oncológicas. ${ }^{13}$ Por ello algunos autores limitan la liberación por debajo de la línea arcuata, para que se pueda colocar una ostomia derecha por debajo en ese punto. ${ }^{13}$

También se han planteado variaciones como el VRAM modificado, que utiliza una isla de piel más pequeña, evitando la necesidad de utilizar una malla para cerrar el defecto de pared abdominal anterior, 10 cual es requerido en un 20 a 30\% de los pacientes, según se describe en los colgajos miocutáneos de recto abdominal. ${ }^{14}$

Se describe una alta incidencia de dispareunia si la pared posterior de la vagina es resecada; algunas series reportan hasta un $50 \%$. A su vez, la capacidad de lograr un orgasmo se reduce hasta en el $50 \%$ de las mujeres. ${ }^{3}$

Es importante destacar que si bien el VRAM tiene una baja incidencia de complicaciones en las múltiples revisiones y se han buscado modificaciones que las reduzcan o eviten, se debe indicar que existen factores que demostraron un aumento en el riesgo de complicaciones del colgajo; se incluyen: obesidad, fumado, antecedente de irradiación, malnutrición e incisiones abdominales transversas. ${ }^{14}$

Se ha determinado que el colgajo VRAM es una herramienta segura para cubrir el defecto en pacientes oncológicos al prevenir ulceración crónica, linfangitis o complicaciones más severas, como ruptura séptica de vasos femorales y desarticulación de cadera. ${ }^{15}$

En general, se concluye que el colgajo VRAM es un método seguro para realizar la reconstrucción pélvica inmediata en pacientes con RAP o exanteración pélvica y radiación, con índices bajos de complicaciones. ${ }^{4}$

\section{Referencias}

1. Barker T, Branagan G, Wright E, Crick A, McGuiness C, Chave H. Vertical rectus abdominis myocutaneous flap reconstruction of the perineal defect after abdominoperineal excision is associated with low morbidity. Colorectal Dis. 2013; 15:1177-83.

2. Sunesen KG, Buntzen S, Tei T, Lindegaard JC, Nørgaard M, Laurberg S. Perineal healing and survival after anal cancer salvage surgery: 10-year experience with primary perineal reconstruction using the vertical rectus abdominis myocutaneous (VRAM) flap. Ann Surg Oncol. 2009; 16:68-77.

3. Corte H, Lefèvre JH, Dehnis N, Shields C, Chaouat M, Tiret E, et al. Female sexual function after abdominoperineal resection for squamous cell carcinoma of the anus and the specific influence of colpectomy and vertical rectus abdominis myocutaneous flap. Colorectal Dis. 2011; 13:774-8.

4. Horch RE, Hohenberger W, Eweida A, Kneser U, Weber K, Arkudas A, et al. A hundred patients with vertical rectus abdominis myocutaneous (VRAM) flap for pelvic reconstruction after total pelvic exenteration. Int J Colorectal Dis. 2014; 29:813-23.

5. Touny A, Othman H, Maamoon S, Ramzy S, Elmarakby H. Perineal reconstruction using pedicled vertical rectus abdominis myocutaneous flap (VRAM). J Surg Oncol. 2014; 110:752-7.

6. Chan S, Miller M, Ng R, Ross D, Roblin P, Carapeti E, et al. Use of myocutaneous flaps for perineal closure following abdominoperineal excision of the rectum for adenocarcinoma. Colorectal Dis. 2010; 12:555-60.

7. Butler CE, Gündeslioglu AO, Rodriguez-Bigas MA. Outcomes of immediate vertical rectus abdominis myocutaneous flap reconstruction for irradiated abdominoperineal resection defects. J Am Coll Surg. 2008; 206:694-703.

8. Lefevre JH, Parc Y, Kernéis S, Shields C, Touboul E, Chaouat M, et al. Abdomino-perineal resection for anal cancer: impact of a vertical rectus abdominis myocutaneus flap on survival, recurrence, morbidity, and wound healing. Ann Surg. 2009; 250:707-11.

9. O'Dowd V, Burke JP, Condon E, Waldron D, Ajmal N, Deasy J, et al. Vertical rectus abdominis myocutaneous flap and quality of life following 


\section{Resección abdominoperineal / Rivera-Chavarría et al}

abdominoperineal excision for rectal cancer: a multi-institutional study. Tech Coloproctol. 2014; 18:901-6.

10. Ho K, Warrier S, Solomon MJ, Lee K. A prepelvic tunnel for the rectus abdominis myocutaneous flap in perineal reconstruction. J Plast Reconstr Aesthet Surg. 2006; 59:1415-9.

11. Howell AM, Jarral OA, Faiz O, Ziprin P, Darzi A, Zacharakis E. How should perineal wounds be closed following abdominoperineal resection in patients post radiotherapy--primary closure or flap repair? Best evidence topic (BET). Int J Surg. 2013; 11:514-7.

12. Di Benedetto G, Siquini W, Bertani A, Grassetti L. Vulvo-perineal reconstruction with a reverse sensitive rectus abdominis salvage flap in a multirecurrent anal carcinoma. J Plast Reconstr Aesthet Surg. 2010; 63:e127-9.
13. Campbell CA, Butler CE. Use of adjuvant techniques improves surgical outcomes of complex vertical rectus abdominis myocutaneous flap reconstructions of pelvic cancer defects. Plast Reconstr Surg. 2011; 128:44758.

14. Berger JL, Westin SN, Fellman B, Rallapali V, Frumovitz M, Ramirez PT, et al. Modified vertical rectus abdominis myocutaneous flap vaginal reconstruction: an analysis of surgical outcomes Gynecol Oncol. 2012; 125:252-5.

15. Daigeler A, Simidjiiska-Belyaeva M, Drücke D, Goertz O, Hirsch T, Soimaru C, et al. The versatility of the pedicled vertical rectus abdominis myocutaneous flap in oncologic patients. Langenbecks Arch Surg. 2011; 396:1271-9. 